\title{
Handling patients with growing small renal masses
}

\author{
Paul Russo, MD, FACS \\ Department of Surgery, Urology Service, Memorial Sloan Kettering Cancer Center, Weill Cornell School of Medicine, New York, NY
}

See related article on page 24 .

Cite as: Can Urol Assoc J 2014;8(1-2):28-9. http://dx.doi.org/10.5489/cuaj.1899

Published online February 10, 2014.

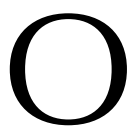
rgan and colleagues evaluate 207 small renal masses in 169 patients in a 5-year multi-institutional follow-up study to assess tumour growth kinetics. ${ }^{1}$ After a median follow-up of 603 days and a median of 5 radiological studies, the median growth rate of the entire cohort was $0.12 \mathrm{~cm} /$ year. An analysis of the cohort using recursive partitioning analysis algorithm did not identify any variables, such as age, symptoms, tumour consistency and maximum diameter at diagnosis that were predictive of growth. In a previous study involving this dataset, the authors demonstrated that growth rate was also not affected by biopsy proof of malignancy and that progression to metastasis occurred in only $1.1 \%$ of patients. ${ }^{2}$ This current study has its limitations, including radiological follow-up using different imaging modalities (computed tomography, magnetic resonance imaging, ultrasonography), which can provide subtle measurement differences when compared, a lack of central radiological review, the use of maximum diameter instead of tumour volume and the absence of precise tumour location (exophytic vs. endophytic or sinus based). Yet, despite these limitations, an important message can be gleaned. Basic clinical factors do not correlate with or predict subsequent tumour growth.

Twenty years ago, all renal tumours, regardless of size, patient ages, and overall medical condition, were treated with radical nephrectomy provided there was a normal appearing contra lateral kidney. However, our current approach to these tumours, particularly the smaller ones, is rapidly changing. Now we understand that renal cortical tumours have complex biology and variable metastatic potential, with nearly $50 \%$ being benign neoplasms or indo- lent malignancies with limited metastatic potential. Benign and indolent renal cortical tumours, such as oncocytoma and chromophobe RCC, can also demonstrate progressive growth and destroy kidneys while reaching massive size, yet the vast majority of these do not metastasize. As in other malignancies, such as breast cancer and soft tissue sarcoma, organ-preserving partial nephrectomy provides the same local tumour control as does radical nephrectomy; at the same time, this operation prevents or delays the onset of chronic kidney disease (CKD) and its potential for subsequent cardiovascular morbidity and mortality. CKD, an underappreciated condition, is highly prevalent in the United States and Canada, with as many as $30 \%$ of renal tumour patients affected at the time of their diagnosis. The creation of preoperative and postoperative prognostic nomograms has better clarified risks and it is now understood that most small renal cortical tumours provide minimal, if any, near term survival threat. A renewed focus on overall survival, life expectancy, and competing medical risks has encouraged strategies of active surveillance (with or without tumour biopsy) in elderly or comorbidly ill patients, nearly one-third of who will die of other causes within 5 years. These important factors are now also incorporated into useful prognostic nomograms.

Although the authors do not identify an exact relationship between tumour growth kinetics and common clinical features that could trigger a therapeutic reaction by the managing urologist, this research effort still provides important counselling tools for us. Small renal tumours in the elderly or otherwise comorbidly ill patients have a slow growth rate and limited metastatic potential. Despite an increasingly technical, expensive and highly marketed armamentarium (i.e., surgical robots, thermal ablation devices), aggressive treatment of such patients is unlikely to yield a true therapeutic dividend; it simply exposes patients to potential surgical complications, inadvertent loss of kidneys or kidney function, unnecessary expense, utilization of critical medi- 
cal resources, and nosocomial infection. These potential adverse events, when taken together, probably provide more of a medical threat than the small renal cortical tumour itself. As the field awaits advanced imaging tools (i.e., malignant tumour specific positron emission tomography imaging) and genomic prognostic assessment of renal biopsies, we urologists still must exercise prudent clinical judgment and careful case selection. For young, healthy patients or those with rapidly growing tumours, surgical intervention sooner rather than later is indicated. For elderly patients or those with serious medical comorbidities, careful surveillance will provide safe short-term care.

Competing interests: Dr. Russo is a protocol consultant with Wilex AG.

\section{References}

1. Organ $M$, Jewett $M$, Basiuk J, et al. Growth kinetics of small renal masses: A prospective analysis from the Renal Cell Carcinoma Consortium of Canada. Can Urol Assoc J 2014;8:24-6. http://dx.doi. org/10.5489/cuaj. 1483

2. Mason RJ, Abdolell M, Trottier $G$, et al. Growth kinetics of renal masses: analysis of a prospective cohort of patients undergoing active surveillance. Eur Urol 2011;59:863-7. http://dx.doi.org/10.1016/i. eururo.2011.02.023

Correspondence: Dr. Paul Russo, Department of Surgery, Urology Service, Memorial Sloan-Kettering Cancer Center, New York, NY; russop@mskcc.org 